\title{
Using Lovász Local Lemma in the space of random injections
}

\author{
Linyuan Lu* László Székely ${ }^{\dagger}$ \\ University of South Carolina $\{1 \mathrm{u}$, szekely $\}$ @math.sc.edu
}

Submitted: Nov 16, 2006; Accepted: Sep 3, 2007; Published: Sep 7, 2007

Mathematics Subject Classification: 05D40, 05A16, 60C05

\begin{abstract}
The Lovász Local Lemma is known to have an extension for cases where independence is missing but negative dependencies are under control. We show that this is often the case for random injections, and we provide easy-to-check conditions for the non-trivial task of verifying a negative dependency graph for random injections. As an application, we prove existence results for hypergraph packing and Turán type extremal problems. A more surprising application is that tight asymptotic lower bounds can be obtained for asymptotic enumeration problems using the Lovász Local Lemma.
\end{abstract}

\section{Introduction}

The Lovász Local Lemma is perhaps one of the most powerful probabilistic tools in combinatorics, which has numerous applications, in addition to combinatorics, in number theory and computer science.

When dependencies of the events are rare, the Lovász Local Lemma provides a general way of proving that with a positive (though tiny) probability, none of the events occur. In some cases an efficient algorithm has been found for finding elements of this tiny event [4]. The main contribution of this paper is to use the Lovász Local Lemma in a space with rich dependencies, in the set of random injections between two sets.

Let $A_{1}, A_{2}, \ldots, A_{n}$ be events in a probability space $\Omega$. A graph $G$ on vertices $[n]$ is called a dependency graph of the events $A_{i}$ 's if $A_{i}$ is mutually independent of all $A_{j}$ with ij $\notin E(G)$.

*This researcher was supported in part by the NSF DMS contract 0701111.

${ }^{\dagger}$ This researcher was supported in part by the NSF DMS contracts 0302307 and 0701111. 
Lemma 1 Lovász Local Lemma (first version) [9] For each $1 \leq i \leq n$, suppose the event $A_{i}$ satisfies $\operatorname{Pr}\left(A_{i}\right) \leq p$, and assume a dependency graph $G$ is associated with these events. Assume that $d$ is an upper bound for the degrees in $G$. If $e(d+1) p<1$, then $\operatorname{Pr}\left(\wedge_{i=1}^{n} \overline{A_{i}}\right)>0$. is positive.

Here is a more general second version, Lemma 2, which implies Lemma 1 by setting $x_{i}=\frac{1}{d+1}$ :

Lemma 2 Lovász Local Lemma (second version) [2] p. 64. Let $A_{1}, \ldots, A_{n}$ be events with dependency graph $G$. If there exist numbers $x_{1}, \ldots, x_{n} \in[0,1)$ such that

$$
\operatorname{Pr}\left(A_{i}\right) \leq x_{i} \prod_{i j \in E(G)}\left(1-x_{j}\right)
$$

for all $i$, then

$$
\operatorname{Pr}\left(\wedge_{i=1}^{n} \overline{A_{i}}\right) \geq \prod_{i=1}^{n}\left(1-x_{i}\right)>0
$$

Going to further generality, a negative dependency graph for $A_{1}, \ldots, A_{n}$ is a simple graph on $[n]$ satisfying

$$
\operatorname{Pr}\left(A_{i} \mid \wedge_{j \in S} \overline{A_{j}}\right) \leq \operatorname{Pr}\left(A_{i}\right)
$$

for any index $i$ and any subset $S \subseteq\{j \mid i j \notin E(G)\}$, if the conditional probability $\operatorname{Pr}\left(\wedge_{j \in S} \overline{A_{j}}\right)$ is well-defined, i.e. $>0$ (in [10], the terminology was lopsidependency graph). We will make use of the fact that Equation (1) trivially holds when $\operatorname{Pr}\left(A_{i}\right)=0$, otherwise the following equation is equivalent to Equation (1):

$$
\operatorname{Pr}\left(\wedge_{j \in S} \overline{A_{j}} \mid A_{i}\right) \leq \operatorname{Pr}\left(\wedge_{j \in S} \overline{A_{j}}\right)
$$

Note that if $A_{i}$ is mutually independent of $A_{j}$ for $j \in S$, then we have

$$
\operatorname{Pr}\left(A_{i} \mid \wedge_{j \in S} \overline{A_{j}}\right)=\operatorname{Pr}\left(A_{i}\right) .
$$

Thus, the dependency graphs always can be considered as negative dependency graphs.

Lemma 3 Lovász Local Lemma (third version) [10], or [2] p. 65. Let $A_{1}, \ldots, A_{n}$ be events with a negative dependency graph $G$. If there exist $x_{1}, \cdots, x_{n} \in[0,1)$ with

$$
\operatorname{Pr}\left(A_{i}\right) \leq x_{i} \prod_{i j \in E(G)}\left(1-x_{j}\right)
$$

for all $i$, then

$$
\operatorname{Pr}\left(\wedge_{i=1}^{n} \overline{A_{i}}\right) \geq \prod_{i=1}^{n}\left(1-x_{i}\right)>0
$$


Note that one easily obtains a version of Lemma 1 for the case of the negative dependency graph from Lemma 3 by setting $x_{i}=\frac{1}{d+1}$. For historical accuracy, [10] described the proof of Lemma 3 in this special setting. The manuscript [14] available on the web gives a detailed proof to Lemma 3. A variant of Lemma 3 has been proved in [1].

The main obstacle for using Lemma 3 is the difficulty to define a useful negative dependency graph other than a dependency graph. In this paper, we will consider the probability space over random injections. Let $U$ and $V$ be two finite sets with $|U| \leq|V|$. Consider the probability space $\Omega=I(U, V)$ of all injections from $U$ to $V$ equipped with a uniform distribution. We are going to provide a criterion for defining the negative dependency graph. We give applications of this criterion in permutation enumeration, hypergraph packing, and Turán type extremal problems.

We do not prove any new result on permutation enumeration, our point is that we are not aware of any previous application of the Lovász Local Lemma in this direction. Our proofs suggest that this possibility is there.

Both for hypergraph packing problems and Turán type extremal problems, the literature mostly focuses on best estimates for particular hypergraphs. Here we give very general bounds that are close to optimal in their general setting, and at the same time, are not very far from the best estimates for particular hypergraphs, when we apply the general setting for them.

For example, for any fixed bipartite graph $G$ on $s$ vertices and any graph $H$ on $n$ vertices, Alon and Yuster [3] proved that for sufficiently large $n, H$ can be covered by vertex-disjoint copies of $G$ if the minimum degree of $H$ is at least $\left(\frac{1}{2}+\epsilon\right) n$ and $s$ divides $n$. We obtain a general (but weaker) result (Theorem 3) on perfect packing problem for any hypergraph $G$.

For Turán type extremal problems, likewise, the literature focuses on particular excluded sub-hypergraphs, like $K_{r+1}^{(r)}$. The few general results available are about the number of edges [16], [12]. Our general results are about excluded sub-hypergraphs, in which every edge meets few other edges, and as before, our estimate is near tight when applied to the well-studied $K_{r+1}^{(r)}$.

The paper is organized as follows. In section 2, we prove our main theorem. We extend the Lovász Local Lemma to the space of random injections by establishing a simple criterion for defining the negative dependency graph. In section 3, we apply our main theorem to asymptotic permutation enumeration. We study the packing problem for any two hypergraphs in section 4 and the perfect packing problem in section 5 . The last application on Turán type extremal problems will be given in section 6 .

In a follow-up paper under preparation, we will show that Lemma 3 applies to the uniform probability space of perfect matchings of $K_{2 n}$ with a proper definition of the negative dependency graph; and we will also show how many of our asymptotically tight lower bounds can be turned actually into an asymptotic formula. 


\section{Main result}

To state our result, we will use the following notations. Every injection from $U$ to $V$ can be viewed as a saturated matching of complete bipartite graph with partite sets $U$ and $V$. In this sense, we define a matching to be a triple $(S, T, f)$ satisfying

1. $S$ is the subset of $U$ and $T$ is a subset of $V$.

2. The map $f: S \rightarrow T$ is a bijection.

We denote the set of all such matchings by $M(U, V)$. Note that the elements of $M(U, V)$ are partial functions from $U$ to $V$ that are injections, and $I(U, V) \subseteq M(U, V)$.

For any permutation $\rho$ of $V$ we define the map $\pi_{\rho}: M(U, V) \rightarrow M(U, V)$ as follows: For any $g \in M(U, V)$ for all $u \in U$

$$
\pi_{\rho}(g)(u)=\rho(g(u))
$$

Clearly for a matching $g_{1}=\left(S_{1}, T_{1}, f_{1}\right)$ if $\pi_{\rho}\left(g_{1}\right)=g_{2}=\left(S_{2}, T_{2}, f_{2}\right)$ then $S_{1}=S_{2}$. Moreover, if $T_{1}$ consists of fixpoints of $\rho$ (i.e. $\rho(v)=v$ for all $v \in T_{1}$ ) then $g_{2}=g_{1}$.

Two matchings $\left(S_{1}, T_{1}, f_{1}\right)$ and $\left(S_{2}, T_{2}, f_{2}\right)$ are said to conflict each other if either " $\exists k \in S_{1} \cap S_{2}, f_{1}(k) \neq f_{2}(k)$ " or " $\exists k \in T_{1} \cap T_{2}, f_{1}^{-1}(k) \neq f_{2}^{-1}(k)$ ". In other words, two matchings do not conflict each other if and only if their union (as a graph) is still a matching.

For a given matching $(S, T, f)$, we define the event $A_{S, T, f}$ as

$$
A_{S, T, f}=\{\sigma \in I(U, V) \mid \sigma(i)=f(i), \forall i \in S\} .
$$

An event $A \in I(U, V)$ is called to be canonical if $A=A_{S, T, f}$ for a matching $(S, T, f)$. Two canonical events conflict each other if their associated matchings conflict. Note that if two events conflict each other, then they are disjoint.

Note that for any permutation $\rho$ of $V$, and any matching $(S, T, f)$, if $\pi_{\rho}((S, T, f))=$ $\left(S, T^{\prime}, f^{\prime}\right)$ then $\pi_{\rho}\left(A_{S, T, f}\right)=A_{S, T^{\prime}, f^{\prime}}$.

We establish a sufficient condition for negative dependency graphs for the space of random injections by showing the following theorem.

Theorem 1 Let $A_{1}, A_{2}, \ldots, A_{n}$ be canonical events in $I(U, V)$. Let $G$ be the graph on $[n]$ defined as

$$
E(G)=\left\{i j \mid A_{i} \text { and } A_{j} \text { conflict }\right\} .
$$

Then $G$ is a negative dependency graph for the events $A_{1}, \ldots, A_{m}$.

Proof: We are supposed to show the inequality (1). If the condition $\wedge_{j \in S} \overline{A_{j}}$ has probability zero, then there is nothing to prove. So assume $\operatorname{Pr}\left(\wedge_{j \in S} \overline{A_{j}}\right)>0$.

By (2), it suffices to show that for any index $i$ and any set $J \subseteq\left\{j: A_{i}\right.$ and $A_{j}$ does not conflict\},

$$
\operatorname{Pr}\left(\wedge_{j \in J} \overline{A_{j}} \mid A_{i}\right) \leq \operatorname{Pr}\left(\wedge_{j \in J} \overline{A_{j}}\right)
$$


For $1 \leq k \leq m$, let $\left(S_{k}, T_{k}, f_{k}\right)$ be the corresponding matching of the event $A_{k}$. We first prove the following claim.

Claim: For any matching $\left(S_{i}, T, f\right)$,

$$
\operatorname{Pr}\left(A_{S_{i}, T, f}\right)=\operatorname{Pr}\left(A_{S_{i}, T_{i}, f_{i}}\right) .
$$

Moreover, if $J \subseteq\left\{j: A_{i}\right.$ and $A_{j}$ does not conflict $\}$, we have

$$
\operatorname{Pr}\left(\left(\wedge_{j \in J} \overline{A_{j}}\right) A_{S_{i}, T, f}\right) \geq \operatorname{Pr}\left(\left(\wedge_{j \in J} \overline{A_{j}}\right) A_{S_{i}, T_{i}, f_{i}}\right) .
$$

Proof of Claim: Fix a matching $\left(S_{i}, T, f\right)$. Let $J^{\prime}$ be the set of indices $j \in J$ so that $A_{j}$ does not conflict $A_{S_{i}, T, f}$. Clearly

$$
\left(\wedge_{j \in J} \overline{A_{j}}\right) A_{S_{i}, T, f}=\left(\wedge_{j \in J^{\prime}} \overline{A_{j}}\right)\left(\wedge_{j \in J \backslash J^{\prime}} \overline{A_{j}}\right) A_{S_{i}, T, f} .
$$

If $j \in J \backslash J^{\prime}$, then $A_{j}$ conflicts to $A_{S_{i}, T, f}$, and so $A_{S_{i}, T, f} \subseteq \overline{A_{j}}$. Therefore

$$
\overline{A_{j}} A_{S_{i}, T, f}=A_{S_{i}, T, f} .
$$

Thus, whether $J \backslash J^{\prime}$ is empty or not, we have

$$
\left(\wedge_{j \in J \backslash J^{\prime}} \overline{A_{j}}\right) A_{S_{i}, T, f}=A_{S_{i}, T, f}
$$

from which it follows that

$$
\left(\wedge_{j \in J} \overline{A_{j}}\right) A_{S_{i}, T, f}=\left(\wedge_{j \in J^{\prime}} \overline{A_{j}}\right) A_{S_{i}, T, f}
$$

Let $\rho: V \rightarrow V$ be a bijection satisfying the following: $\rho(v)=v$ for any $v \in \cup_{j \in J^{\prime}} T_{j}$ and for $w \in T, \rho(w)=f_{i}\left(f^{-1}(w)\right)$. By the definition of $J^{\prime}$ we have that for each $j \in J^{\prime}$ if $u \in S_{i} \cap S_{j}$ then $f(u)=f_{i}(u)=f_{j}(u)$, therefore such a $\rho$ clearly exists. Moreover, for each $j \in J^{\prime}, T_{j}$ consists of fixpoints of $\rho, \rho(T)=T_{i}$, and for $u \in S_{i}, \rho(f(u))=f_{i}(u)$.

This implies that $\pi_{\rho}\left(\left(S_{i}, T, f\right)\right)=\left(S_{i}, T_{i}, f_{i}\right)$, from which equation (6) follows. Also for each $j \in J^{\prime}$ we have $\pi_{\rho}\left(\left(S_{j}, T_{j}, f_{j}\right)\right)=\left(S_{j}, T_{j}, f_{j}\right)$. Thus, for each $j \in J^{\prime}$

$$
\pi_{\rho}\left(\overline{A_{j}} A_{S_{i}, T, f}\right)=\overline{A_{j}} A_{S_{i}, T_{i}, f_{i}},
$$

from which

$$
\pi_{\rho}\left(\left(\wedge_{j \in J^{\prime}} \overline{A_{j}}\right) A_{S_{i}, T, f}\right)=\left(\wedge_{j \in J^{\prime}} \overline{A_{j}}\right) A_{S_{i}, T_{i}, f_{i}}
$$

Using equations (8) and (10) we obtain

$$
\begin{aligned}
\operatorname{Pr}\left(\left(\wedge_{j \in J} \overline{A_{j}}\right) A_{S_{i}, T, f}\right) & \left.=\operatorname{Pr}\left(\left(\wedge_{j \in J^{\prime}} \overline{A_{j}}\right) A_{S_{i}, T, f}\right)\right) \\
& =\operatorname{Pr}\left(\left(\wedge_{j \in J^{\prime}} \overline{A_{j}}\right) A_{S_{i}, T_{i}, f_{i}}\right) \\
& \geq \operatorname{Pr}\left(\left(\wedge_{j \in J} \overline{A_{j}}\right) A_{S_{i}, T_{i}, f_{i}}\right) .
\end{aligned}
$$

The proof of the claim is finished. 
For the fixed set $S_{i}$, the collection of events $\left\{A_{S_{i}, T, f} \mid\left(S_{i}, T, f\right)\right.$ is a matching $\}$ forms a partition of the space $\Omega=I(U, V)$.

From this partition and equations (6) and (7) we get

$$
\begin{aligned}
\operatorname{Pr}\left(\wedge_{j \in J} \overline{A_{j}}\right) & =\sum_{\left(S_{i}, T, f\right)} \operatorname{Pr}\left(\left(\wedge_{j \in J} \overline{A_{j}}\right) A_{S_{i}, T, f}\right) \\
& \geq \sum_{\left(S_{i}, T, f\right)} \operatorname{Pr}\left(\left(\wedge_{j \in J} \overline{A_{j}}\right) A_{S_{i}, T_{i}, f_{i}}\right) \\
& =\sum_{\left(S_{i}, T, f\right)} \operatorname{Pr}\left(\wedge_{j \in J} \overline{A_{j}} \mid A_{S_{i}, T_{i}, f_{i}}\right) \operatorname{Pr}\left(A_{S_{i}, T_{i}, f_{i}}\right) \\
& =\sum_{\left(S_{i}, T, f\right)} \operatorname{Pr}\left(\wedge_{j \in J} \overline{A_{j}} \mid A_{S_{i}, T_{i}, f_{i}}\right) \operatorname{Pr}\left(A_{S_{i}, T, f}\right) \\
& =\operatorname{Pr}\left(\wedge_{j \in J} \overline{A_{j}} \mid A_{S_{i}, T_{i}, f_{i}}\right) .
\end{aligned}
$$

\section{Asymptotic Permutation Enumeration}

There is a well-known asymptotic formula for the number of fixed-point-free permutations of $n$ elements (or derangements of $n$ elements), $n ! / e$. Surprisingly, the Lovász Local Lemma gives this asymptotic formula as lower bound. Let us be given a set $U$ of $n$ elements.

To apply Theorem 1 , set $V=U$, for $i \in U$ set $S_{i}=T_{i}=\{i\}$, define $f_{i}: S_{i} \rightarrow T_{i}$ by $i \mapsto i$. Set $A_{i}=A_{S_{i}, T_{i}, f_{i}}$ and observe that $A_{i}$ consists of permutations that fix $i$. We will use empty negative dependency graph, i.e. $E(G)=\emptyset$.

For the purposes of Lemma 3 select $x_{i}=1 / n$. This choice is allowed, as $\operatorname{Pr}\left(A_{i}\right)=1 / n$ and the product in $(3)$ is empty. The conclusion is that $\operatorname{Pr}\left(\wedge_{i} \overline{A_{i}}\right) \geq\left(1-\frac{1}{n}\right)^{n}$, and this number converges to $1 / e$.

Going further, it is known that the probability of a random permutation not having any $k$-cycle is asymptotically $e^{-1 / k}[5]$. With some effort, we can get this as a lower bound for the probability from Lovász Local Lemma. Let us be given a set $U$ of $n$ elements. To apply Theorem 1 , set $V=U$, for $I \subset U,|I|=k$ set $S_{I}=T_{I}=I$; and consider all $f_{I}^{\alpha}: I \rightarrow I$ functions $(\alpha=1,2, \ldots,(k-1) !)$ that correspond to a $k$-cycle on $I$.

Define the event $A_{I, \alpha}=A_{I, I, f_{I}^{\alpha}}$. The vertices of the negative dependency graph will be the $\left(\begin{array}{l}n \\ k\end{array}\right)(k-1)$ ! events, and we join $A_{I, \alpha}$ with $A_{J, \beta}$ if $I \cap J \neq \emptyset$. Every degree in the negative dependency graph is bounded by $\left[\left(\begin{array}{c}n \\ k\end{array}\right)-\left(\begin{array}{c}n-k \\ k\end{array}\right)\right](k-1) !=\frac{(n)_{k}}{k}-\frac{(n-k)_{k}}{k}$, where $(n)_{k}$ is the falling factorial notation. For the purposes of Lemma 3 select $x_{I, \alpha}=x=\frac{1+\frac{c_{k}}{n}}{(n)_{k}}$, Note that $\operatorname{Pr}\left(A_{I, \alpha}\right)=\frac{1}{(n)_{k}}$, and a little calculation shows that

$$
\frac{1}{(n)_{k}} \leq x(1-x)^{\frac{(n)_{k}}{k}-\frac{(n-k)_{k}}{k}}
$$


by the definition of $x$, if $c_{k}$ is sufficiently large, due to the fact that $\frac{(n)_{k}}{k}-\frac{(n-k)_{k}}{k}=O\left(n^{k-1}\right)$. (Just take the logarithm of both sides of (11) that we need to prove and expand the logarithms into series.) Hence, (3), the condition of Lemma 3, holds. The conclusion of Lemma 3 is that $\operatorname{Pr}\left(\wedge_{I, \alpha} \overline{A_{I, \alpha}}\right) \geq\left(1-\frac{1+\frac{c_{k}}{n}}{(n)_{k}}\right)^{\left(\begin{array}{l}n \\ k\end{array}\right)(k-1) !}$, and this number on the right hand side converges to $e^{-1 / k}$.

Let us turn now to the enumeration of Latin rectangles. An $k \times n$ Latin rectangle is a sequence of $k$ permutations of $\{1,2, \ldots, n\}$ written in a matrix form, such that no column has any repeated entries. Let $L(k, n)$ denote the number of $k \times n$ Latin rectangles. The current best asymptotic formula [11] for $L(k, n)$ works for $k=o\left(n^{6 / 7}\right)$. Without going into details of the history of the problem, the previous best range was $k=o\left(n^{1 / 2}\right)$, with the use of the Chen-Stein method [7], [19], showing

$$
L(k, n) \sim(n !)^{k} e^{-\left(\begin{array}{c}
k \\
2
\end{array}\right)-\frac{k^{3}}{6 n}} .
$$

Formula (12) has had an unexpected proof [17], where the inequality

$$
(n !)^{k} \prod_{r=1}^{k-1}\left(1-\frac{r}{n}\right)^{n} \leq L(k, n)
$$

which was proved from the van der Waerden inequality for the permanent, provided the lower bound for the asymptotic formula.

Our goal now is to show (13) from Lemma 3 for $k=o\left(n^{1 / 2}\right)$. We need the following very general lemma.

Lemma 4 Assume that $G$ is a negative dependency graph for the events $A_{1}, A_{2}, \ldots, A_{n}$. Assume further that $V(G)$ has a partition into classes, such that any two events in the same class have empty intersection. For any partition class $J$, let $B_{J}=\vee_{j \in J} A_{j}$. Now the quotient graph of $G$ is a negative dependency graph for the events $B_{J}$.

Proof. We have to show that if $\mathcal{K}$ is a subset of non-neighbors of $J$ in the quotient graph, then $\operatorname{Pr}\left(B_{J} \mid \wedge_{K \in \mathcal{K}} \overline{B_{K}}\right) \leq \operatorname{Pr}\left(B_{J}\right)$. By the additivity of (conditional) probability over mutually exclusive events, it is sufficent to show that

$$
\operatorname{Pr}\left(A_{j} \mid \wedge_{K \in \mathcal{K}} \overline{B_{K}}\right) \leq \operatorname{Pr}\left(A_{j}\right)
$$

holds for every $j \in J$. However, $\wedge_{K \in \mathcal{K}} \overline{B_{K}}=\wedge_{i \in \cup \mathcal{K}} \overline{A_{i}}$, and every $i \in \cup \mathcal{K}$ is a non-neighbor of $j$ in $G$, according to the definition of the quotient graph. Therefore, (14) holds as $G$ is a negative dependency graph.

Let us select now $k$ permutations $\pi_{1}, \pi_{2}, \ldots, \pi_{k}$ of the elements $\{1,2, \ldots, n\}$ randomly and independently, and fill in the entries $\pi_{i}(j)$ into a $k \times n$ matrix. We want to give a lower bound for the probability that the first $t+1$ rows make a Latin rectangle under the condition that first $t$ rows make a Latin rectangle. Fix an arbitrary $t \times n$ Latin rectangle now with rows $\pi_{1}, \pi_{2}, \ldots, \pi_{t}$. Define the event $A_{i j}$ by $\pi_{i}(j)=\pi_{t+1}(j)$. These are 
canonical events. Let $G$ be the graph whose vertices are the $(i, j)$ entries for $j=1,2, \ldots, n$, $i=1,2, \ldots, t$, and every $\left(i_{1}, j\right)$ is joined with every $\left(i_{2}, j\right)$. The maximum degree in this graph is $t-1=o\left(n^{1 / 2}\right)$. With the choice $x_{i j}=2 / n$ these events satisfy (3) in the graph $G$, and therefore the graph $G$ according to Theorem 1 is a negative dependency graph. Define the events $B_{j}=\vee_{1 \leq i \leq t} A_{i j}$. Clearly $\operatorname{Pr}\left(B_{j}\right) \leq t / n$. The quotient graph is empty, and is a negative dependency graph for the $B_{j}$ events. Lemma 3 applies and $\operatorname{Pr}\left(\wedge_{j=1}^{n} \overline{B_{j}}\right) \geq(1-t / n)^{n}$. Iterating this estimate, formula (13) follows.

Spencer made a joke in [18], that Lovász Local Lemma 1 can prove the existence of an injection from an $a$-element set into a $6 a$-element set, while the naive approach requires a $\Theta\left(a^{2}\right)$ size codomain, as it is well-known from the 'Birthday Paradox'. Now using Lemma 3 in combination with Lemma 5 below, we can show that a random function from an $a$-element set into an $a$-element set is an injection with probability at least $\left(\frac{1}{e}-o(1)\right)^{a}$, giving a combinatorial proof to a weakened Stirling formula! (Apart from Lemma 4, this is the only result in the paper not using Theorem 1.)

We say that the events $A_{1}, A_{2}, \ldots, A_{n}$ are symmetric, if the probability of any boolean expression of these sets do not change, if we substitute $A_{\pi(i)}$ to the place of $A_{i}$ simultaneously, for any permutation $\pi$ of $[n]$.

Lemma 5 Assume that the events $A_{1}, A_{2}, \ldots, A_{n}$ are symmetric, and let $p_{i}$ denote $\operatorname{Pr}\left(\overline{A_{1}} \wedge\right.$ $\left.\overline{A_{2}} \wedge \cdots \wedge \overline{A_{i}}\right)$ for $i=1,2, \ldots, n$ and let $p_{0}=1$. If the sequence is logconvex, i.e. $p_{k}^{2} \leq$ $p_{k-1} p_{k+1}$ for $k=1,2, \ldots, n-1$, then Lemma 3 applies with an empty negative dependency graph, i.e. with $x_{i}=p_{1}$.

Proof. Mathematical induction on the number of terms in the condition yields that $\operatorname{Pr}\left(A_{1} \mid \overline{A_{2}} \wedge \overline{A_{3}} \wedge \ldots \wedge \overline{A_{k}}\right)=1-p_{k} / p_{k-1} \geq 1-p_{k+1} / p_{k}=\operatorname{Pr}\left(A_{1} \mid \overline{A_{2}} \wedge \overline{A_{3}} \wedge \ldots \wedge \overline{A_{k}} \wedge \overline{A_{k+1}}\right)$.

Consider a set $A$ with $|A|=a$ and a set $B$ with $|B|=b$, and assume $a \leq b$. Consider a random function $f$ from $A$ to $B$. For $u \in A$, define the event $A_{u}=$ the value $f(u)$ occurs with multiplicity 2 or higher. The events $A_{u}$ are symmetric. Clearly $\operatorname{Pr}\left(A_{u}\right)=1-b(b-$ $1)^{a-1} / b^{a}$. Direct calculation shows that $p_{i}=\operatorname{Pr}\left(\overline{A_{u_{1}}} \wedge \overline{A_{u_{2}}} \cdots \overline{A_{u_{i}}}\right)=i !\left(\begin{array}{c}b \\ i\end{array}\right)(b-i)^{a-i} / b^{a}$. The logconvexity of the $p_{i}$ sequence is algebraically equivalent to $(b-k)^{2 a-2 k-1} \leq(b-$ $k+1)^{a-k}(b-k-1)^{a-k-1}$ for $k=1, \ldots, a-1$. In the case $a=b$, set $n=a-k$, and the last inequality is algebraically equivalent to the well-known fact $\left(1+\frac{1}{n-1}\right)^{n-1} \leq\left(1+\frac{1}{n}\right)^{n}$ for $n \geq 2$, while the case $n=1$ corresponds to $p_{a-1}^{2} \leq p_{a-2} p_{a}$, which is easy to check. Hence, using Lemma 3, we obtain that the probability that a random $A \rightarrow A$ function is an injection, is at least $\left(1-p\left(A_{1}\right)\right)^{a}=(1-1 / a)^{a(a-1)}=\left(\frac{1}{e}-o(1)\right)^{a}$, pretty close to the correct asymptotics $a ! / a^{a}=\sqrt{2 \pi a} e^{-a}(1+o(1))$.

\section{Packing problem}

A hypergraph $H$ consists of a vertex set $V(H)$ together with a family $E(H)$ of subsets of $V(H)$, which are called edges of $H$. A $r$-uniform hypergraph, or $r$-graph, is a hypergraph whose edges have the same cardinality $r$. The complete $r$-graph on $n$ vertices is denoted by $K_{n}^{(r)}$. 
Packing problem of hypergraphs: For two $r$-uniform hypergraphs $H_{1}, H_{2}$, and an integer $n \geq \max \left\{\left|V\left(H_{1}\right)\right|,\left|V\left(H_{2}\right)\right|\right\}$, are there injections $\phi_{i}: V\left(H_{i}\right) \rightarrow[n]$, for $i=1,2$ such that $\phi_{1}\left(H_{1}\right)$ and $\phi_{2}\left(H_{2}\right)$ are edge-disjoint?

Theorem 2 For $i=1,2$, assume that $H_{i}$ is an $r$-uniform hypergraph with $m_{i}$ edges, such that every edge in $H_{i}$ intersects at most $d_{i}$ other edges of $H_{i}$. If $\left(d_{1}+1\right) m_{2}+\left(d_{2}+1\right) m_{1}<$ $\frac{1}{e}\left(\begin{array}{l}n \\ r\end{array}\right)$, then there exist injections of $V\left(H_{1}\right)$ and $V\left(H_{2}\right)$ into $K_{n}^{(r)}$ such that the natural images of $H_{1}$ and $H_{2}$ are edge-disjoint.

Proof: Without loss of generality, we assume that $H_{2}$ is given as a sub-hypergraph of $K_{n}^{(r)}$. Consider a random injection of $V\left(H_{1}\right)$ into $V\left(K_{n}^{(r)}\right)$; this injection extends to $E\left(H_{1}\right)$ in the natural way. Our probability space will be $I(U, V)$ with $U=V\left(H_{1}\right)$ and $V=[n]$. Consider two edges $F_{1}$ (of $H_{1}$ ) and $F_{2}$ (of $H_{2}$ ); and a bijection $\phi: F_{1} \rightarrow F_{2}$. The events $A_{F_{1}, F_{2}, \phi}$ will be our bad events. We have

$$
\operatorname{Pr}\left(A_{F_{1}, F_{2}, \phi}\right)=\frac{1}{r !\left(\begin{array}{l}
n \\
r
\end{array}\right)}=\frac{1}{(n)_{r}} .
$$

Let $G$ be the negative dependency graph of those $A_{F_{1}, F_{2}, \phi}$ events. An event $A_{F_{1}, F_{2}, \phi}$ conflicts another event $A_{F_{1}^{\prime}, F_{2}^{\prime}, \phi^{\prime}}$ if and only if

1. Edges $F_{1}$ and $F_{1}^{\prime}$ have empty intersection while their images $F_{2}$ and $F_{2}^{\prime}$ have nonempty intersection.

2. Edges $F_{1}$ and $F_{1}^{\prime}$ have non-empty intersection but $\phi$ and $\phi^{\prime}$ are defined differently in some intersection point.

An event $A_{F_{1}, F_{2}, \phi}$ can have at most $r !\left(d_{2}+1\right) m_{1}-1$ conflicts of the first type, and at most $r$ ! $\left(d_{1}+1\right) m_{2}$ conflicts of the second type, thus the maximal degree $d$ in the negative dependency graph is at most

$$
r !\left[\left(d_{1}+1\right) m_{2}+\left(d_{2}+1\right) m_{1}\right]-1 .
$$

Apply Lemma 1 in the negative dependency graph setting. With positive probability, all bad events $A_{f_{1}, f_{2}, \phi}$ can be avoided simultaneously if

$$
e(d+1) \operatorname{Pr}\left(A_{f_{1}, f_{2}, \phi}\right)<1 .
$$

Remark: The constant coefficient $\frac{1}{e}$ in Theorem 2 can not be replaced by 2 as shown by the following example.

Let $r=2$ and $H_{1}$ be the graph on $n=s(s-1)$ vertices consisting of $s-1$ vertexdisjoint copies of the complete graph $K_{s}$; and let $H_{2}$ be the graph on $n=s(s-1)$ vertices consisting of a single $K_{s}$ and $n-s$ isolated vertices. The complement graph $\overline{H_{1}}$ is $K_{s}$-free by the pigeonhole principle. (In fact, $\bar{H}$ is the maximum $K_{s}$-free graph on $n$ vertices by Turán theorem [21].) Therefore, copies of $H_{1}$ and $H_{2}$ can not be packed into $K_{n}$ with 
disjoint edge sets. In this example, we have $d_{1}=d_{2}=2(s-2)$ and $m_{1}=(s-1)\left(\begin{array}{l}s \\ 2\end{array}\right)$, $m_{2}=\left(\begin{array}{l}s \\ 2\end{array}\right)$. It is easy to see that Therefore, we have

$$
\begin{aligned}
\left(d_{1}+1\right) m_{2}+\left(d_{2}+1\right) m_{1} & =(2 s-3)(s-1)\left(\begin{array}{l}
s \\
2
\end{array}\right)+(2 s-3)\left(\begin{array}{l}
s \\
2
\end{array}\right) \\
& =(2 s-3)\left(\begin{array}{l}
s \\
2
\end{array}\right)<2\left(\begin{array}{c}
s(s-1) \\
2
\end{array}\right)=2\left(\begin{array}{l}
n \\
2
\end{array}\right) .
\end{aligned}
$$

Here the last inequality holds for all $s \geq 2$ by an easy calculation.

\section{$5 \quad$ Perfect Packing}

For two $r$-uniform hypergraphs, $H$ and $G$, we say that $H$ has a perfect $G$-packing if there exist sub-hypergraphs $G_{1}, \ldots, G_{k}$ of $H$, each isomorphic to $G$, such that the vertex sets $V\left(G_{1}\right), \ldots, V\left(G_{k}\right)$ partition $V(H)$.

A necessary condition for the existence of perfect $G$-packing is that $|V(H)|$ is divisible by $|V(G)|$. We will prove the following theorem.

Theorem 3 Suppose that two r-uniform hypergraphs $G$ and $H$ satisfy the following.

1. $G$ has s vertices, $H$ has $n$ vertices, and $n$ is divisible by $s$.

2. $G$ has $m$ edges, and each edge in $G$ intersects at most d other edges of $G$.

3. For any vertex $v$ of $H$, the degree of $v$ in $H$ is at least $(1-x)\left(\begin{array}{l}n-1 \\ r-1\end{array}\right)$.

If $x<\frac{1}{e\left(d+1+r^{2} \frac{m}{s}\right)}$, then then $H$ has a perfect $G$-packing.

A special case is that $G$ is the $r$-graph with a single edge. We have $m=1, d=0$, and $s=r$.

Corollary 1 Suppose the degree of each vertex in an $r$-graph $H$ on $n$ vertices is at least $\left(1-\frac{1}{e(r+1)}\right)\left(\begin{array}{l}n-1 \\ r-1\end{array}\right)$. If $n$ is divisible by $r$, then $H$ has a perfect matching.

Proof of Theorem 3: Let $H_{1}$ be the union of $\frac{n}{s}$ vertex-disjoint copies of $G$ and $\bar{H}$ be the complement graph of $H$. Observe that $H$ has a perfect $G$-packing if and only if $H_{1}$ and $H_{2}=\bar{H}$ can be packed into $K_{n}^{(r)}$. Now we apply Theorem 2 . Notice that $d_{1}=d$ and $m_{1}=\left|E\left(H_{1}\right)\right|=\frac{n}{s}|E(G)|=\frac{n m}{s}$. The degree of any vertex in $\bar{H}$ is at most $x\left(\begin{array}{l}n-1 \\ r-1\end{array}\right)$ by the third condition. We have

$$
d_{2} \leq r x\left(\begin{array}{c}
n-1 \\
r-1
\end{array}\right)-r+1 \leq r x\left(\begin{array}{l}
n-1 \\
r-1
\end{array}\right)-1 .
$$

It suffices to have

$$
\left(d_{1}+1\right) m_{2}+\left(d_{2}+1\right) m_{1} \leq(d+1) x\left(\begin{array}{l}
n \\
r
\end{array}\right)+\left(r x\left(\begin{array}{c}
n-1 \\
r-1
\end{array}\right)\right) \frac{n m}{s} \leq \frac{1}{e}\left(\begin{array}{l}
n \\
r
\end{array}\right) .
$$

THE EleCtronic Journal of COMBinatorics 13 (2007), \#R63 
The second inequality is equivalent to

$$
x<\frac{1}{e\left(d+1+\frac{r^{2} m}{s}\right)} .
$$

as desired.

\section{Turán type extremal problems}

For a fixed $r$-graph $G$, let $t(n, G)$ denote the smallest integer $m$ such that every $r$ uniform hypergraph on $n$ vertices with $m+1$ edges must contain a copy of $G$. The limit $\lim _{n \rightarrow \infty} \frac{t(n, G)}{\left(\begin{array}{l}n \\ r\end{array}\right)}$ always exists [13]. We denote it by $\pi(G)$. We have

Theorem 4 Suppose each edge in an r-graph $G$ intersects at most d other edges. Then we have

$$
\pi(G) \leq 1-\frac{1}{(d+1) e}
$$

Proof: Consider an $r$-graph $H$ on $n$ vertices and $m=t(n, G)-1$ edges, which do not contain a copy of $G$. Note that each edge of $\bar{H}$ can intersect at most $r\left(\begin{array}{c}n-1 \\ r-1\end{array}\right)-r+1$ other edges. The conclusion of Theorem 2 does not apply to $G$ and $\bar{H}$. Therefore we must have

$$
(d+1)\left(\left(\begin{array}{l}
n \\
r
\end{array}\right)-m\right)+\left(r\left(\begin{array}{l}
n-1 \\
r-1
\end{array}\right)-r+2\right)|E(G)| \geq \frac{1}{e}\left(\begin{array}{l}
n \\
r
\end{array}\right),
$$

and by rearranging terms

$$
\begin{aligned}
\frac{m}{\left(\begin{array}{c}
n \\
r
\end{array}\right)} \leq 1 & -\frac{1}{e(d+1)}+\frac{r^{2}}{n} \frac{|E(G)|}{d+1} \\
& =1-\frac{1}{e(d+1)}+O\left(\frac{1}{n}\right)
\end{aligned}
$$

as desired as $n \rightarrow \infty$ while $d$ and $r$ are fixed.

The general upper bound for Turán density in term of the number of edges is first obtained by Sidorenko [16]

$$
\pi(G) \leq 1-\frac{1}{f-1}
$$

for any $r$-graph $G$ with $f$ edges. It has slightly been improved by Keevash [12] to

$$
\pi(G)<1-\frac{1}{f-1}-(1+O(1))\left(2 r !^{2 / r} f^{3-2 / r}\right)^{-1}
$$

for fixed $r \geq 3$ and $f \rightarrow \infty$.

If a hypergraph $G$ is a clique, in which any pair of edges have non-empty intersection, then we have $d=f-1$. Inequality (16) is closer to the best known upper bound. For 
example, consider a special complete $r$-graph $K_{r+1}^{(r)}$ on $r+1$ vertices, de Caen [6], Sidorenko ([15]), Tazawa and Shirakura [20] proved

$$
1-\frac{(1+o(1)) \ln r}{2 r} \leq \pi\left(K_{r+1}^{(r)}\right) \leq 1-\frac{1}{r}
$$

The upper bound was improved [8] to

$$
\pi\left(K_{r+1}^{(r)}\right) \leq 1-\frac{5 r+12-\sqrt{9 r^{2}+24 r}}{2 r(r+3)}=1-\frac{1}{r}-\frac{1}{r^{2}}+O\left(\frac{1}{r^{3}}\right)
$$

for odd $r$.

Theorem 4 only gives

$$
\pi\left(K_{r+1}^{(r)}\right) \leq 1-\frac{1}{e(r+1)}
$$

It is still quite comparable to those best known upper bounds for $\pi\left(K_{r+1}^{(r)}\right)$ except for the constant coefficient $e$.

However, for hypergraph $G$ with less intersection, (say, $d<\frac{f}{e}$ ) inequality (15) often offers a much better upper bound on $\pi(G)$ than inequality (16) does.

Acknowledgment: We thank Eva Czabarka for her suggestions at writing this paper.

\section{References}

[1] M. Albert, A. Frieze, B. Reed, Multicolored Hamiltonian cycles, Electronic J. Combinarorics 2 (1995) \#R10, pp. 1-13.

[2] N. Alon, J. H. Spencer, The Probabilistic Method, second edition, John Wiley and Sons, New York, 2000.

[3] N. Alon and R. Yuster, H-factors in dense graphs, J. Comb. Theory, Ser. B 66 (1996), 269-282.

[4] J. Beck, An algorithmic approach to the Lovász local lemma. I., Random Structures and Algorithms 2 (1991) 343-365.

[5] D. Borkowitz, The name game: exploring random permutations, Mathematics Teacher 98 (2005) (October)

and its appendix http://faculty. wheelock.edu/dborkovitz/articles/ngm6.htm

[6] D. de Caen, Extension of a theorem of Moon and Moser on complete subgraphs, Ars Comb. 16 (1983): 5-10.

[7] L. Chen, Poisson approximation for independent trials, Ann. Probab. 3 (1975), 534545. 
[8] F. Chung and L. Lu, An upper bound for the Turán number $t_{3}(n, 4), J$. Combin. Theory Ser. A 87(2), (1999), 381-389.

[9] P. Erdős and L. Lovász, Problems and results on 3-chromatic hypergraphs and some related questions, in Infinite and Finite Sets, A. Hajnal et. al., Eds., Colloq. Math. Soc. J. Bolyai 11, North Holland, Amsterdam, 1975, 609-627.

[10] P. Erdős and J. H. Spencer, Lopsided Lovász local lemma and latin transversals, Discrete Appl. Math. 30 (1991), 151-154.

[11] C. D. Godsil, B. D. McKay, Asymptotic enumeration of Latin rectangles, J. Comb. Theory B 48 (1990), 19-44.

[12] P. Keevash, The Turán problem for hypergraphs of fixed size, Electronic J. Combinatorics 12 (2005), \#N11.

[13] G. O. H. Katona, T. Nemetz, M. Simonovits, On a problem of Turán in the theory of graphs, (in Hungarian) Mat. Lapok 15 (1964), 228-238.

[14] C. Y. Ku, Lovász local lemma, http://www .maths .qmul.ac.uk/ cyk/

[15] A. Sidorenko, The method of quadratic forms in the combinatorial problem of Turán, Moscow Univ. Math. Bull. 37 No. 1 (1982), 1-5.

[16] A. Sidorenko, Extremal combinatorial problems in spaces with continuous measure, Issled. Operatsiui ASU 34 (1989), 34-40.

[17] I. Skau, A note on the asymptotic number of Latin rectangles, Europ. J. Combinatorics 19 (1998), 617-620.

[18] J. H. Spencer, Ten Lectures on the Probabilistic Method, CBMS 52, SIAM, 1987.

[19] C. Stein, Asymptotic evaluation of the number of Latin rectangles, J. Comb. Theory A 25 (1978), 38-49.

[20] S. Tazawa and T. Shirakura, Bounds on the cardinality of clique-free family in hypergraphs, Math. Sem. Notes Kobe Univ. 11 (1983), 277-281.

[21] P. Turán, On an extremal problem in graph theory. Mat. Fiz. Lapok. 48 (1941): 436452 (in Hungarian). English translation in Collected Papers of Paul Turán (P. Erdős, ed.), 231-240. Budapest: Akadémiai Kiadó, 1990. 